\title{
Optimum Design of Thin Walled Tube on the Mechanical Performance of Super Stud Bolt*
}

\author{
Yang XIAO**, Nao-Aki NODA**, Masahiro KUHARA**, Kinjiro SAITO***, \\ Masato NAGAWA $* * * *$ and Atsushi YUMOTO***** \\ **Department of Mechanical Engineering, Kyushu Institute of Technology, \\ 1-1 Sensui-cho, Tobata-ku, Kitakyushui, Fukuoka, 804-0001 Japan \\ ***Nittetsu Yawata Engineering Co., Ltd., \\ 2-2 Tobihata-cho, Tobata-ku, Kitakyushui, Fukuoka, 804-0001 Japan \\ ****Daiki Kogyo Co., Ltd. \\ 11-16 Hama-cho, moji-ku, Kitakyushui, Fukuoka, 801-0856 Japan \\ *****Nittetsu Plant Designing Corp. \\ 46-59 DAiji Nakabaru, Tobata-ku, Kitakyushui, Fukuoka, 804-0002 Japan \\ E-mail: noda@mech.kyutech.ac.jp
}

\begin{abstract}
The bolts and nuts are widely used in various fields as important joining elements with long history. However, screw loosening is easily induced by vibration and external loads, and sometimes causes very serious accident without notice. In this paper, a special stud bolt named "Super Stud Bolt (SSB)" is studied, which can prevent loosening effectively. Between the upper threads and the lower threads, there is a thin walled tube which can be deformed along the axial direction so that the phase difference is designed and SSB is developed. During fastening, this phase difference induces the contrary forces on the surfaces of the upper threads and the lower threads, which bring out the anti-loosening performance. In this study, both courses of processing and fastening are simulated, and the relationship between axial force and displacement is illustrated with the finite element method. In this analysis, the large plastic strains and elastic-plastic large deformation theory are applied to 4 nodes axial symmetric FEM models. Through the comparison among the results, the desirable range of the phase difference of lower and upper threads is decided. Since the thin walled tube is the heart of anti-loosening mechanism, optimum original dimensions are discussed in this study.
\end{abstract}

Key words: Plastic Working, Machine Element, Press Working, Anti-loosening, Thin Walled Tube, Prevailing Torque

\section{Introduction}

Threaded connections play a critical role in engineering with long history. Because of their relatively low cost and easy disassembly for maintenance, they are widely used for mechanical products and structures in modern times. However, self-loosening, which is one of the most frequent failure modes for threaded connections, brings out the failure of engineering products and several serious accidents. Therefore, much attention has been paid to the research on this anti-loosening.

${ }^{*}$ Received 8 Feb., 2010 (No. T2-07-1093)

Japanese Original : Trans. Jpn. Soc. Mech. Eng., Vol. 74, No. 743, A (2008), pp.954-960 (Received 10 Dec., 2007) [DOI: 10.1299/jmmp.4.1455]

Copyright $\odot 2010$ by JSME

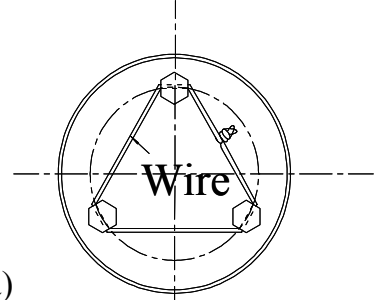

(b)

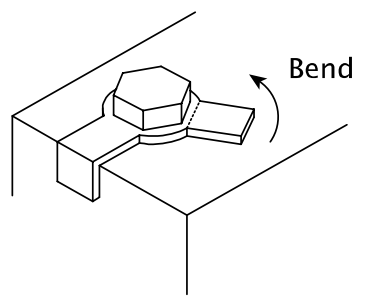

Fig. 1 Anti-loosening stud bolts previously proposed 


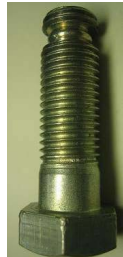

(a) Photo

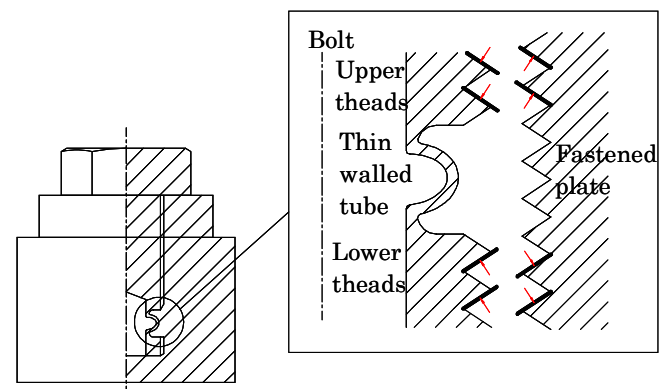

(b) Anti-loosening theory

Fig. 2 Super stud bolt recently proposed

In recent years, several special nuts have been invented in order to prevent self-loosening ${ }^{(1)-(4)}$. However, it is difficult to improve the anti-loosening capability of the stud bolt connections. Figure 1 shows examples of anti-loosening stud bolts; one is bolt with wire, the other is plant bend. However, they cannot be loosened easily and cannot be used repeatedly, once they are fastened. In order to make up for these disadvantages, the anti-loosening bolt, the super stud bolt $(\mathrm{SSB})^{(5)}$ is invented as shown in Fig. 2.

\section{Anti-loosening principle of the Super Stud Bolt}

Super stud bolt can prevent self-loosening, so that it can be used easily to save time and labor. Anti-loosening principle is shown in Fig. 2(b). As shown in Fig. 2(b), the thin walled tube between the upper threads and the lower threads plays an important role for the anti-loosening mechanism. The thin walled tube can be deformed along the axis, and the phase difference of lower and upper threads is designed in the course of processing. Accordingly, the deformation must be produced in order that the threads meet each other in the course of fastening. Then, the thread contact force in Fig. 2(b) comes out due to this deformation. This force is called the griping force, by which the prevailing torque is produced, so that the self-loosening can be prevented.

In this paper, the capability of the anti-loosening is investigated using FEM, and the best dimensions is discussed. Both courses of processing and fastening are simulated, and the relation between axial force and displacement is studied. Through the comparison for anti-loosening capability, the reasonable range of the phase difference of lower and upper threads is decided. Since the thin walled tube is the heart of anti-loosening mechanism, the best original dimensions are also discussed in this study.

\section{Notation}

In this paper, the following notations are used.

$L, L_{1} \sim L_{5} \quad$ Length of the thin walled tube defined in Figs. 5 and $6(\mathrm{~mm})$

$R$ Curvature radius of the thin walled tube defined in Fig. $3(\mathrm{~mm})$

$t$ Thickness of the thin walled tube defined in Fig. $3(\mathrm{~mm})$

$z \quad$ Forced displacement defined in Fig. $3(\mathrm{~mm})$

$P \quad$ Pitch of nut (mm)

$b, b^{\prime} \quad$ Spring back length defined in Figs. 5 and $6(\mathrm{~mm})$

$\alpha, \alpha_{1} \sim \alpha_{5} \quad$ Phase difference of lower and upper threads defined in Figs. 5 and $6(\mathrm{~mm})$

$h, h_{1} \sim h_{5} \quad$ Height of nut defined in Figs. 5 and $6(\mathrm{~mm})$

$\sigma_{z} \quad$ Axial stress $(\mathrm{MPa})$

$\varepsilon_{z} \quad$ Axial strain

$K$ Spring constant $(\mathrm{N} / \mathrm{m})$

$T_{s} \quad$ Prevailing torque $(\mathrm{N} \cdot \mathrm{m})$

\section{Analysis method and model}

\subsection{Analysis method}

Using the axi-symmetric FE model, both courses of processing and fastening-loosening 
of SSB are simulated. The processing course is shown in Fig. 3, and the course of fastening-loosening is shown in Fig. 4. In Fig. 3 (a), the length $L$ of thin walled tube is produced on the top of normal bolt by machining. At this time, the phase difference of lower and upper threads is zero. As shown in Fig. 3 (b), the thin walled tube is pressed by $(p-\alpha)$ $\mathrm{mm}$. Here, $\alpha(\mathrm{mm})$ is the nominal phase difference between lower and upper threads, which is much smaller than the pitch of bolt $(p=2 \mathrm{~mm})$. When the press is removed, spring back happens as shown in Fig. 3 (c). Here, $L_{1}=L-(p-\alpha)$ and $L_{2}=L-(p-\alpha)+b$, so that the real phase difference of lower and upper threads $\alpha_{2}=\alpha+b=p-\left(L-L_{2}\right)$ appears. Figure 4 (d) is the same as Fig. 3 (c), and Fig. 4 (e) shows the phase difference of lower and upper threads becomes 0 because the threads of the bolt and nut can meet each other while the nut is fastened. In Fig. 4 (f), spring back $b^{\prime}$ happens after loosening. In order to simulate both courses, elastic-plastic large deformation theory will be applied in this analysis. The value of $L, L_{1} \sim L_{5}, P, \alpha_{1} \sim \alpha_{5}$ is shown in parentheses of Figs. 3 and 4.

The material of the SSB is SCM435 (JIS), whose stress-strain relation in Fig. 5 is used in the elastic-plastic finite element analysis of the thin walled tube. Here, Young's modulus is $206 \mathrm{GPa}$, Poison's ratio is 0.3 , and the yielding stress is $800 \mathrm{MPa}$.

\subsection{Analysis model}

As shown in Fig. 6 (a), the SSB is simplified into an axi-symmetric model to be studied. Here the bottom is fixed, while the forced displacement $z(\mathrm{~mm})$ is placed on the top of the model by the rigid body. In Fig. 6 (a) dimensions are changed as $L=4.8 \sim 6.8 \mathrm{~mm}, R=$ $0.5 \sim 1.0 \mathrm{~mm}, t=0.4 \sim 0.8 \mathrm{~mm}$, and the effects of the shape are examined.

In this study, using a commercial FEM code, MSC.Marc, the thin walled tube is mainly studied with the finite element model as Fig. 6 (b) shows. The details of thin walled tube elements are shown in Fig. 6 (c). The four nodes axisymmetric FEM models and the elastic-plastic large strain theories are applied. Total number of elements is 2082 and nodes number is 2243. Both courses of processing and fastening-loosening of SSB are simulated by pressing and unloading FEM models as shown in Fig. 6.

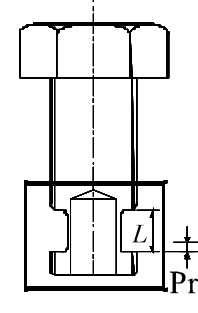

(a) Before de forming $(L=5.8 \mathrm{~mm})$

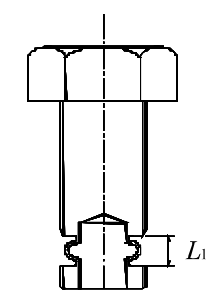

$p-\alpha$

$L_{1}=L-(p-\alpha)$

$\alpha_{1}=\alpha$

(b) Loading and deforming

( $L_{1}=3.9 \mathrm{~mm}, L-L_{1}=1.9 \mathrm{~mm}$, $\left.p=2 \mathrm{~mm}, \alpha_{1}=\alpha=0.1 \mathrm{~mm}\right)$

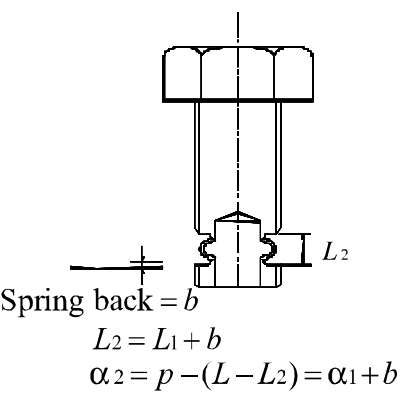

(c) After unloading

$\left(L_{2}=3.96 \mathrm{~mm}, L-L_{2}=1.84 \mathrm{~mm}\right.$, $b=0.06 \mathrm{~mm}, \alpha_{2}=0.16 \mathrm{~mm}$ )

Fig. 3 Dimension change in the course of processing

[( ): Values in Fig. 8]

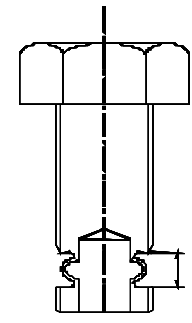

$L_{3}=L_{2}$ $\alpha_{3}=\alpha_{2}$

(d) Before fastening

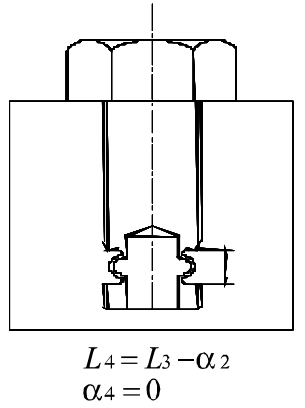

(e) Fastening

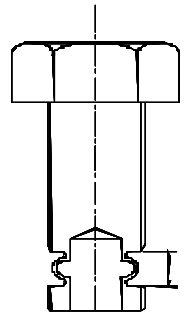

$L_{5}=L_{4}+b^{\prime}$ $\alpha_{5}=p-\left(L-L_{5}\right)$

(f) After loosening

$\begin{array}{lll}\left(L_{3}=3.96 \mathrm{~mm}, L-L_{3}=1.84 \mathrm{~mm},\right. & \left(L_{4}=3.8 \mathrm{~mm}, L-L_{4}=2.0 \mathrm{~mm},\right. & \left(L_{5}=3.86 \mathrm{~mm}, L-L_{5}=1.94 \mathrm{~mm},\right. \\ \left.\alpha_{4}=0.16 \mathrm{~mm}\right) & \left.\alpha_{4}=0 \mathrm{~mm}\right) & \left.\alpha_{5}=0.06 \mathrm{~mm}, b^{\prime}=0.16 \mathrm{~mm}\right)\end{array}$

$\begin{array}{lll}\left(L_{3}=3.96 \mathrm{~mm}, L-L_{3}=1.84 \mathrm{~mm},\right. & \left(L_{4}=3.8 \mathrm{~mm}, L-L_{4}=2.0 \mathrm{~mm},\right. & \left(L_{5}=3.86 \mathrm{~mm}, L-L_{5}=1.94 \mathrm{~mm}\right. \\ \left.\alpha_{4}=0.16 \mathrm{~mm}\right) & \left.\alpha_{4}=0 \mathrm{~mm}\right) & \left.\alpha_{5}=0.06 \mathrm{~mm}, b^{\prime}=0.16 \mathrm{~mm}\right)\end{array}$

Fig. 4 Dimension change in the course of fastening

[( ): Values in Fig. 8] 


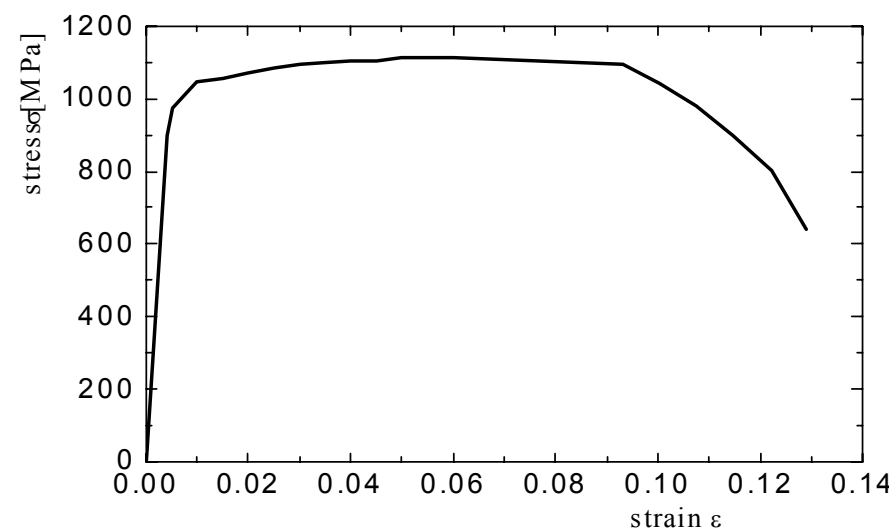

Fig. 5 Relation between stress and strain for SCM435

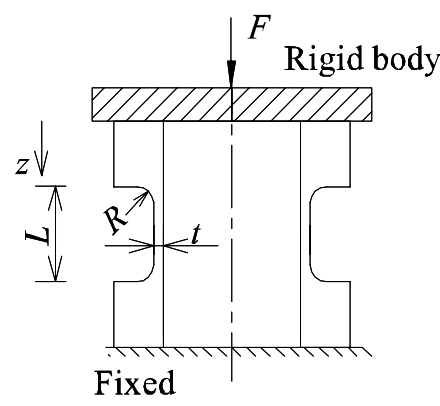

Fig. 6 (a) Analysis model

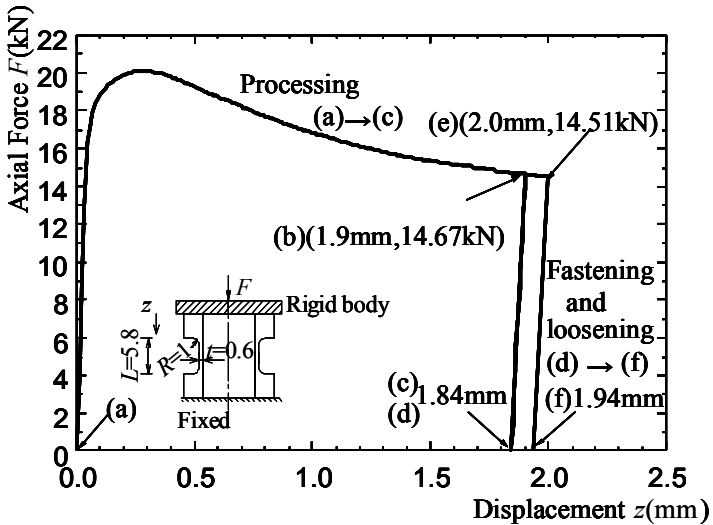

(a) $\rightarrow$ (c) Processing

$(\mathrm{d}) \rightarrow$ (e) Fastening

$(\mathrm{e}) \rightarrow$ (f) Loosening

(f) $\rightarrow$ (e) Fastening from second time

(e) $\rightarrow$ (f) Loosening from second time

Fig. 7 Relation between axial force and displacement $(L=5.8 \mathrm{~mm}, R=1 \mathrm{~mm}, t=0.6 \mathrm{~mm})$

\section{Analysis results}

\subsection{The relationship between axial force and displacement}

A standard model M16 nut is employed, and the dimension of the thin walled tube is shown in Fig.6(a), here, $L=5.8 \mathrm{~mm}, R=1 \mathrm{~mm}, t=0.6 \mathrm{~mm}$ and $\mathrm{p}-\alpha=1.9 \mathrm{~mm}$. The relation between axial force and displacement is shown in Fig.7. The course of processing is indicated as the line $(\mathrm{a}) \rightarrow(\mathrm{b}) \rightarrow(\mathrm{c})$. In Fig. 8 , normal bolt $(\mathrm{p}=2.0 \mathrm{~mm})$ is pressed by $L-L_{1}=1.9 \mathrm{~mm}$ (point b) and the current phase difference is $\alpha=\alpha_{1}=0.1 \mathrm{~mm}$. However, due to spring back $b=0.06 \mathrm{~mm}$, the plastic deformation is $L-L_{2}=1.84 \mathrm{~mm}$ at the course of processing and the real phase difference of lower and upper threads is $\alpha_{2}=\alpha_{1}+b=0.16 \mathrm{~mm}$. The line from (d) to (e) shows the course of fastening, and the line from (e) to (f) shows the course of loosening. In those loading and unloading courses, the plastic deformation occurs 
and the permanent deformation $L_{3}-L_{5}=1.94-1.84=0.10 \mathrm{~mm}$ occurs. Then, only small phase difference of lower and upper threads $(2.00-1.94=0.06 \mathrm{~mm})$ remains. However, in consecutive fastening-loosening courses, the relation between the axial force and the displacement moves to the line from (f) to (e). Because the deformation in this course is totally elastic, plastic deformation does not occur and therefore the phase difference of lower and upper threads $0.06 \mathrm{~mm}$ would not be changed anymore.

\subsection{The anti-loosening capability}

In this study, anti-loosening capability is estimated by prevailing torque $T_{s}$. The prevailing torque is the fastening torque when the bottom surface of the bolt head does not contact with the upper surface of the clamped body. The value of $T_{s}$ can be evaluated by Eq.(1), which is usually used for evaluating the fastening torque for normal bolt and nut ${ }^{(6)}$. This method was proposed in previous study ${ }^{(7)}$ and it is confirmed that the values of Eq.(1) are in good agreement with experimental results. It is also known that loosening can be prevented if prevailing torque is more than $7.3 \mathrm{kN}$ in the experiment ${ }^{(8)}$.

When the threads meet each other in Fig. 7(e), the axial force is obtained as $14.51 \mathrm{kN}$ from FEM. Therefore we can substitute this value into Eq.(1), and the prevailing torque may be evaluated as $T_{s}=20.64 \mathrm{~N} \cdot \mathrm{m}$.

$$
T=\frac{d_{2}}{2} F \tan \rho^{\prime}+\frac{d_{2}}{2} F \tan \beta+\frac{d_{w}}{2} \mu_{w} F
$$

$$
\begin{aligned}
& \text { Where } \quad T \text { is fastening torque, }(\mathrm{N} \cdot \mathrm{m}) \\
& F \text { is axial force, }(\mathrm{kN}) \\
& d \text { is the diameter of screw, and approximately } \\
& d_{w} \text { is effective contact diameter between the head of bolt and the } \\
& \text { clamp. here, } d_{w}=0 \\
& d_{2} \text { is effective diameter } d_{2}=0.92 d \\
& \rho^{\prime} \text { is friction angle between the threads, here } \tan \left(\rho^{\prime}\right)=0.15 \\
& \beta \text { is lead angle of threads, } 2.4796 \text { (deg) } \\
& \mu_{w} \text { is average friction coefficient on the interface between the head } \\
& \text { of bolt and the clamp. }
\end{aligned}
$$

\subsection{Anti-loosening capability under various phase differences of lower and upper threads}

Since the phase difference of lower and upper threads of the SSB controls the capability of anti-loosening, the effect of the phase difference on the spring constant is studied, and suitable dimensions are discussed.

If the axial force is too small, the anti-loosening capability is little; on the other hand, while the axial force is too large, fastening becomes difficult. Therefore, there is a certain range of the suitable axial force $\Delta F$, which can prevent self-loosening effectively (see Fig. 8). In Fig. 8, when the spring constant is $k_{l}$, the range of the phase difference of lower and

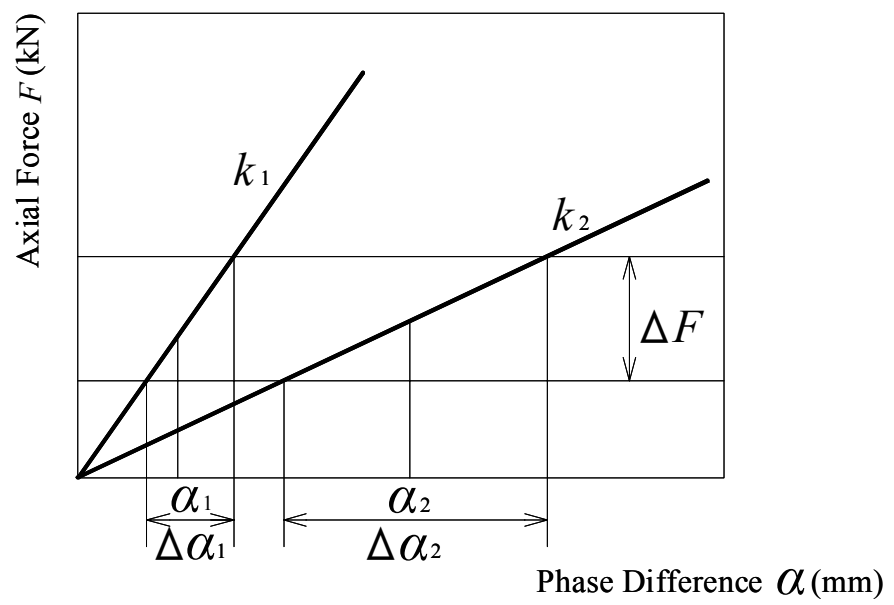

Fig. 8. Relation between the axial force and phase difference of lower and upper threads 


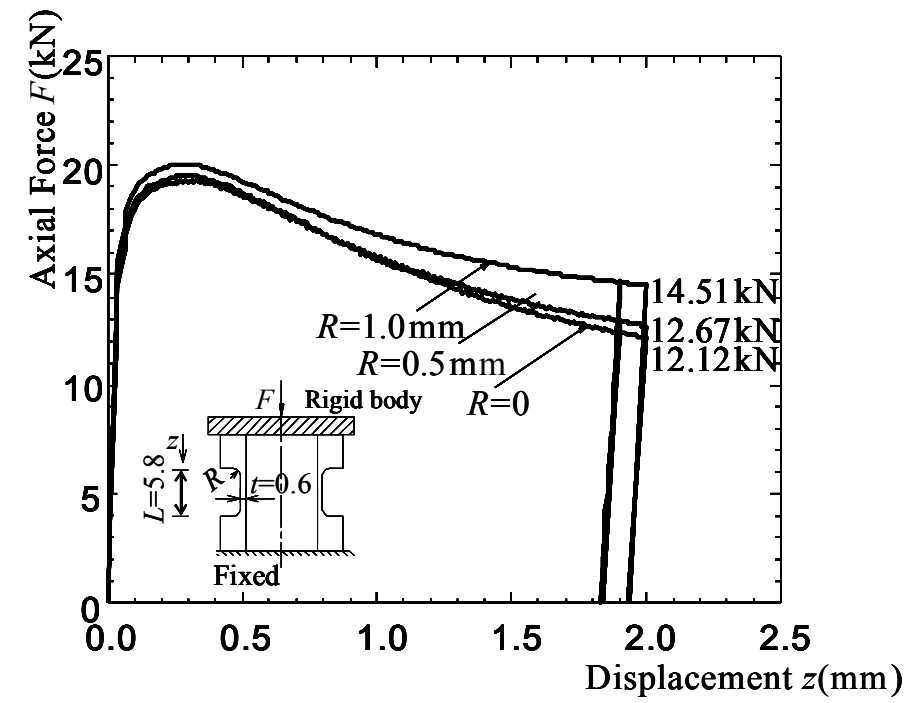

Fig. 9 Relation between axial force and displacement for different curvatures $R(L=5.8 \mathrm{~mm}, t=0.6 \mathrm{~mm})$

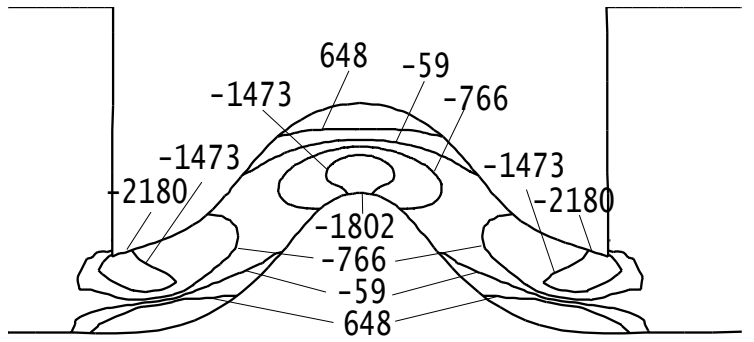

(a) $R=0 \mathrm{~mm}$

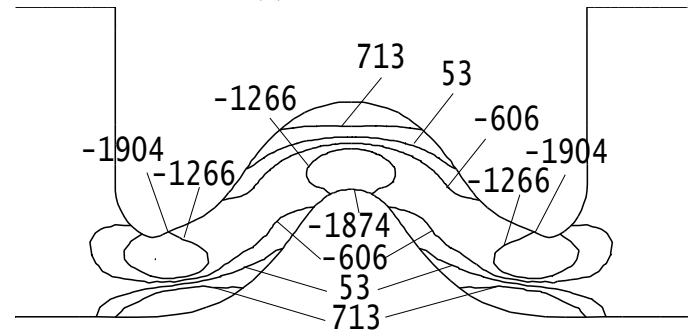

(b) $R=0.5 \mathrm{~mm}$

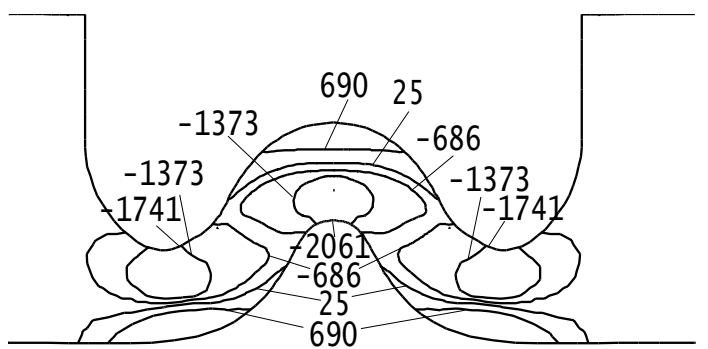

(c) $R=1.0 \mathrm{~mm}$

Fig. 10 Stress distribution $\sigma_{z}(\mathrm{MPa})$ for different curvatures $R$

$$
\left(L=5.8 \mathrm{~mm}, t=0.6 \mathrm{~mm}, \alpha_{1}=0.1 \mathrm{~mm}\right)
$$

upper threads $\alpha_{1}$ should be in $\Delta \alpha_{1}$; while the spring constant is $k_{2}<k_{1}$, the range of the phase difference of lower and upper threads $\alpha_{2}$ should be in $\Delta \alpha_{2}$; then, $\Delta \alpha_{1}<\Delta \alpha_{2}$. That is to say, the suitable range of the phase difference of lower and upper threads becomes wider if spring constant is smaller ${ }^{(7)}$. Usually of SSB includes some error of the phase difference in the course of processing, and therefore small spring constants may be desirable.

In Table 1, initial dimensions of SSB are fixed as $R=1.0 \mathrm{~mm}$, and $t=0.6 \mathrm{~mm}$ and $L-L_{1}$ 
$=1.9 \mathrm{~mm}$ in Fig. 3 . In order to attain various phase differences of lower and upper threads, the length of the thin tube $L$ is changed from $4.8 \mathrm{~mm}$ to $6.8 \mathrm{~mm}$.

\section{Effect of the dimension of the thin walled tube on the anti-loosening capability}

The thin walled tube is the heart of SSB because the dimension has large effect on the anti-loosening capability, stress distribution and spring constant. In this chapter, the inference of the dimensions $R, L, t$ in Fig. 6 (a) are investigated, and the best dimensions are examined.

\subsection{Effect of curvature radius $R$}

In order to improve stress distribution at both ends of thin walled tube, the effect of curvature radius $R$ in Fig. 6 (a) is studied. As shown in Fig. 6 (a), $L$ and $t$ are fixed as $L=$ $5.8 \mathrm{~mm}, t=0.6 \mathrm{~mm}$, and $R$ is changed. When the press displacement is 1.9 , the relation between axial force and displacement is shown in Fig. 9. From Fig. 9, when $L$ and $t$ are fixed, It is seen that the curvature radius has little effect on the anti-loosening capability and spring constant ${ }^{(9)}$.

When $R$ is $0.0,0.5$, and 1.0, the stress distributions of the thin walled tube in Fig. 6 (b) are shown in Fig. 10 (a), (b) and (c), respectively. While $R$ is smaller, the stress concentration is more serious at both ends of the thin walled tube. Conversely, while $R$ is larger, at the center of the thin walled tube, the stress becomes larger. The stress distribution is the most desirable in Fig. 10 (b) while $R$ is $0.5 \mathrm{~mm}$.

\subsection{Effect of length of the thin walled tube $L$}

To investigate the effect of length $L$, dimensions $R$ and $t$ are fixed as $R=0.5 \mathrm{~mm}$, $t=0.6 \mathrm{~mm}$, and $L$ is changed in Fig. 6 (a). When $L$ is $4.8 \sim 6.8$ and press displacement is $1.9 \mathrm{~mm}$ and current difference is $\alpha_{1}=0.1 \mathrm{~mm}$, the results are shown in Table 1 and Fig. 11 .

As Table 1 shows, while $L$ is longer, the spring constant becomes smaller, and spring back $b$ in Fig. 3 becomes larger and the real phase difference $\left(\alpha_{2}=\alpha_{1}+b\right)$ also increases.

Table 1 Effect of initial length of thin tube $L$

\begin{tabular}{c|c|c|c}
$\left(L-L_{1}=1.9 \mathrm{~mm}\right.$ in Fig.3, $R=1.0 \mathrm{~mm}, t=0.6 \mathrm{~mm}$ in Fig.6(a) \\
\hline $\begin{array}{c}\text { Length of thin walled } \\
\text { tube } L(\mathrm{~mm})\end{array}$ & $\begin{array}{c}\text { Spring } \\
\text { constant } \\
k\left(\times 10^{8} \mathrm{~N} / \mathrm{m}\right)\end{array}$ & $\begin{array}{c}\text { Spring back } \\
\text { (Elastic deformation) } \\
b(\mathrm{~mm})\end{array}$ & $\begin{array}{c}\text { Prevailing torque } \\
T s(\mathrm{~N}-\mathrm{m})\end{array}$ \\
\hline 4.8 & 4.36 & 0.04 & 25.08 \\
5 & 3.88 & 0.04 & 23.86 \\
5.8 & 2.63 & 0.06 & 20.64 \\
6 & 2.42 & 0.06 & 19.83 \\
6.8 & 1.89 & 0.07 & 17.73 \\
\hline
\end{tabular}

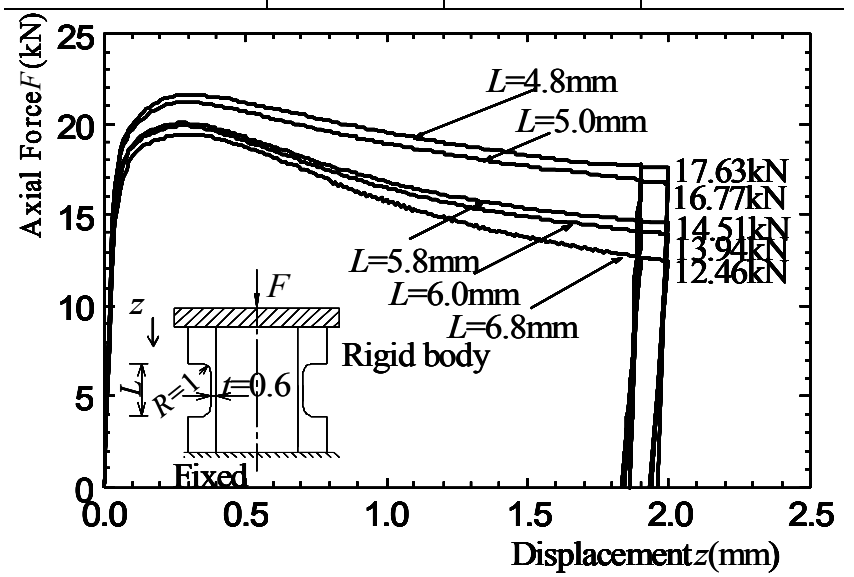

Fig. 11 Relation between axial force and displacement for different initial lengths

$$
(R=1.0 \mathrm{~mm}, t=0.6 \mathrm{~mm})
$$


Here, spring back $b$ may be regarded as an amount of elastic deformation. It should be noted that this elastic deformation is much smaller than the phase difference of lower and upper threads $(0.1 \sim 0.15 \mathrm{~mm})$. In other words, large plastic deformation always exists in the course of first fastening-loosening shown in Fig. 11 when $L$ is $4.8 \sim 6.8 \mathrm{~mm}$. From Fig.10, it is seen that axial force decreases with the increase of $L$, so that the prevailing torque becomes smaller. That is to say, the anti-loosening capability becomes lower as $L$ is longer. In addition, the cost of processing is higher when $L$ becomes longer. According to the above, it may be concluded that $L=4.8 \mathrm{~mm}$ is the best. The length of thin walled tube should be designed as short as possible if the thin walled tube can be deformed as show in Fig. 5 . While $L<4.8 \mathrm{~mm}$, the length of straight part of thin tube $(L-2 R)$ is very short, so that it is difficult to make the swelled part.

\subsection{Effect of thickness of the thin walled tube $t$}

In order to improve both processing and fastening courses, the effect of the thickness of the thin walled tube $t$ is studied in this section. While the $L$ and $R$ are fixed, thickness $t$ is changed as $0.4,0.6$, and $0.8 \mathrm{~mm}$. The results are shown in Table 2 . The spring back becomes less when $t$ increases. It means elastic deformation is larger and errors of phase difference become wider with the decrease of thickness $t$.

In Fig. 12, relation between axial force and displacement for different thicknesses $t$ are shown. When $t=0.4 \mathrm{~mm}$, the maximum axial forces at both courses of processing and fastening are the smallest. That is to say, both courses of processing and fastening become easier when thickness $t$ is thinner.

The stress distributions $\sigma_{z}$ are indicated in Fig. 13 (a), (b) and (c) and the distributions of the plastic strain $\varepsilon_{z}$ are shown in Fig. 14. From Figs. 13 and 14, it is seen that the stress and strain distribution becomes complicated with increasing thickness $t$. The stress distribution of $t=0.4 \mathrm{~mm}$ is desirable, and therefore, it may be concluded that the best thickness $t$ is $0.4 \mathrm{~mm}$.

Table 2 Effect of initial thickness of thin walled tube $t$

$$
(R=1.0 \mathrm{~mm}, L=5.8 \mathrm{~mm})
$$

\begin{tabular}{c|c|c|c}
\hline $\begin{array}{c}\text { Thickness of thin } \\
\text { walled tube } t(\mathrm{~mm})\end{array}$ & $\begin{array}{c}\text { Spring constant } \\
k\left(\times 10^{8} \mathrm{~N} / \mathrm{m}\right)\end{array}$ & $\begin{array}{c}\text { Spring back } \\
(\text { Elastic deformation) } \\
b(\mathrm{~mm})\end{array}$ & $\begin{array}{c}\text { Prevailing } \\
\text { torque } \\
T s(\mathrm{~N}-\mathrm{m})\end{array}$ \\
\hline 0.8 & 5.37 & 0.05 & 36.98 \\
0.6 & 2.63 & 0.06 & 20.64 \\
0.4 & 0.77 & 0.07 & 7.75 \\
\hline
\end{tabular}

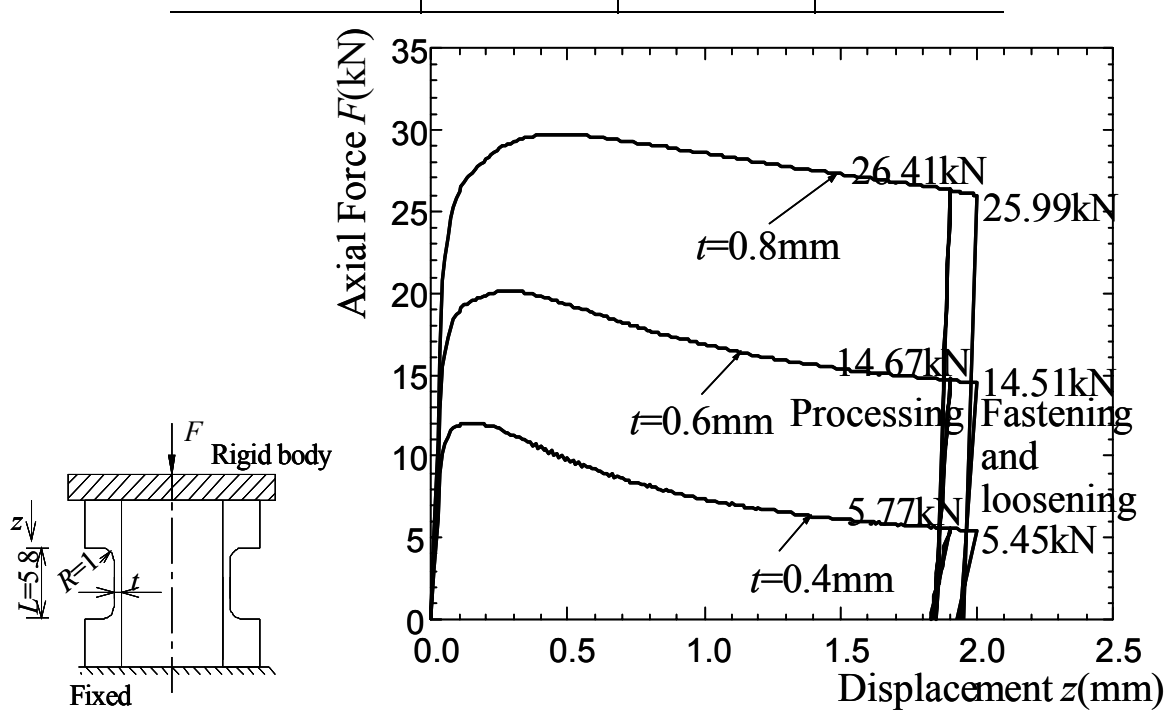

Fig. 12. Relation between axial force and displacement for different thicknesses $t$ ( $R=1.0 \mathrm{~mm}, L=5.8 \mathrm{~mm})$ 


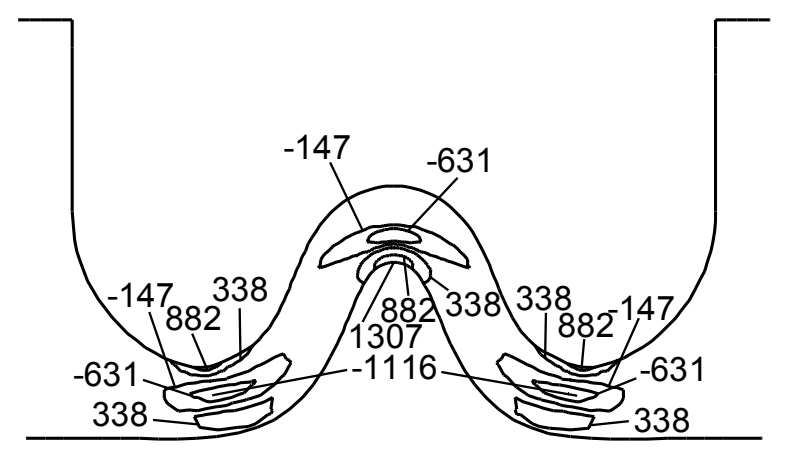

(a) $t=0.4 \mathrm{~mm}$

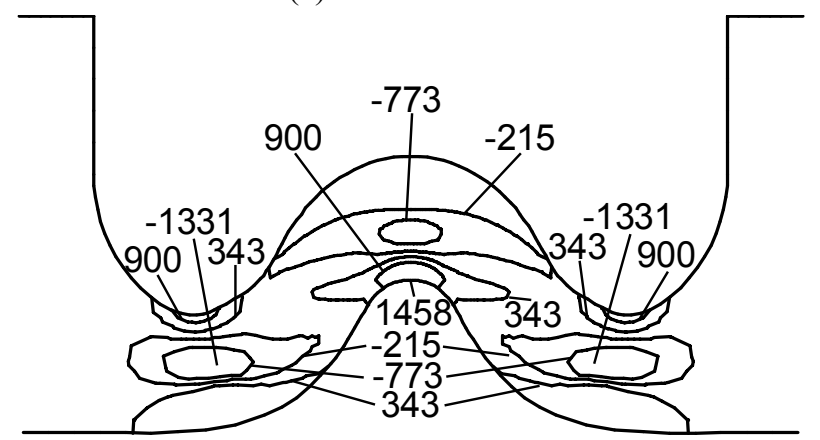

(b) $t=0.6 \mathrm{~mm}$

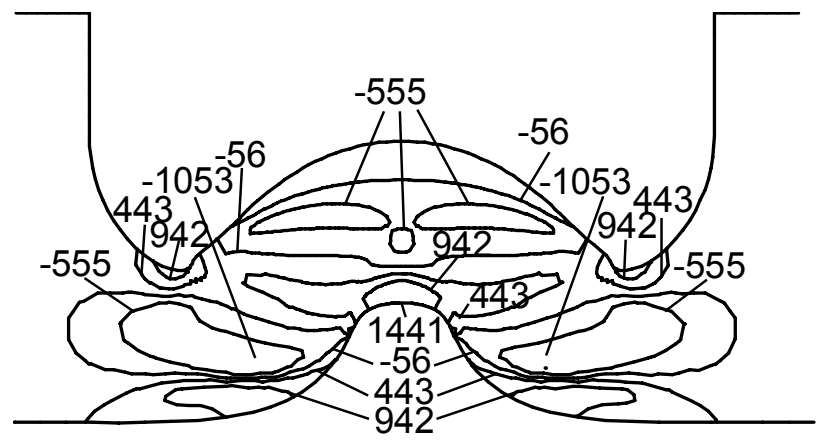

(c) $t=0.8 \mathrm{~mm}$

Fig. 13 Distribution of $\sigma_{z}(\mathrm{MPa})(R=1.0 \mathrm{~mm}, L=5.8 \mathrm{~mm})$

\subsection{The best dimensions}

According to the above discussion, the best dimensions of M16 SSB are designed as $R$ $=0.5 \mathrm{~mm}, L=4.8 \mathrm{~mm}$, and $t=0.4 \mathrm{~mm}$. The relation between axial force and displacement is shown in Fig. 15. In this case, the spring constant is $k=8.22 \times 10^{7} \mathrm{~N} / \mathrm{m}$ which is less than the values in Tables 1 and 2. On the other hand, the spring back $b=0.07 \mathrm{~mm}$ and it is larger, so that the elastic strain is larger. The prevailing torque $T_{s}=7.91 \mathrm{~N} \cdot \mathrm{m}$ and it is enough to prevent the loosening ${ }^{(10)}$. The stress distributions $\sigma_{z}$ and the distributions of the plastic strain $\varepsilon_{z}$ are shown in Figs. 16 and 17, respectively.

\subsection{The best phase difference}

Phase difference is very important to the anti-loosening capability of SSB. The pressing displacement at the course of processing is change from $1.7 \sim 2.0 \mathrm{~mm}$, so that current phase difference is $0.3 \sim 0 \mathrm{~mm}$, relation between axial force and displacement under various phase difference is shown in Fig. 18. It is seen that the spring constant is almost equal when the phase difference of lower and upper threads is changed. Also, the prevailing torque is nearly constant when the phase difference of lower and upper threads is larger than 0.10 . 


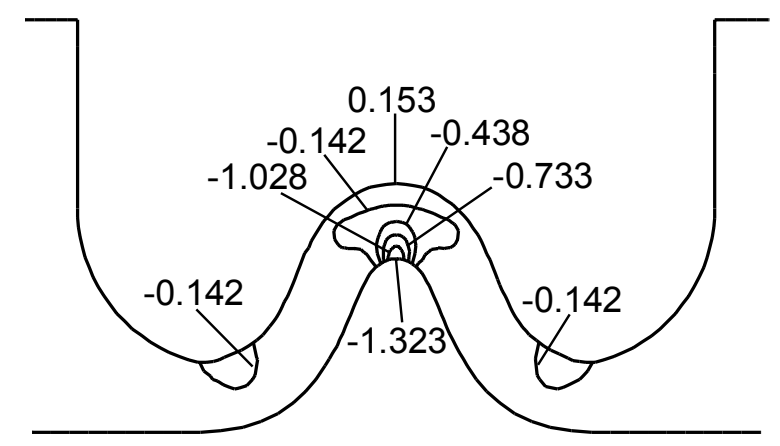

(a) $t=0.4 \mathrm{~mm}$

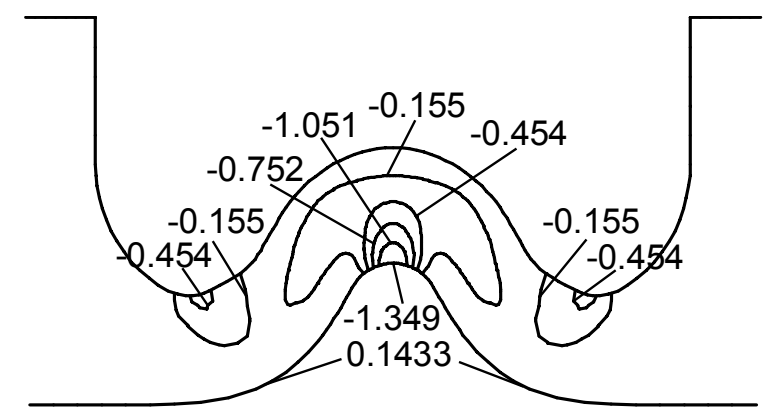

(b) $t=0.6 \mathrm{~mm}$

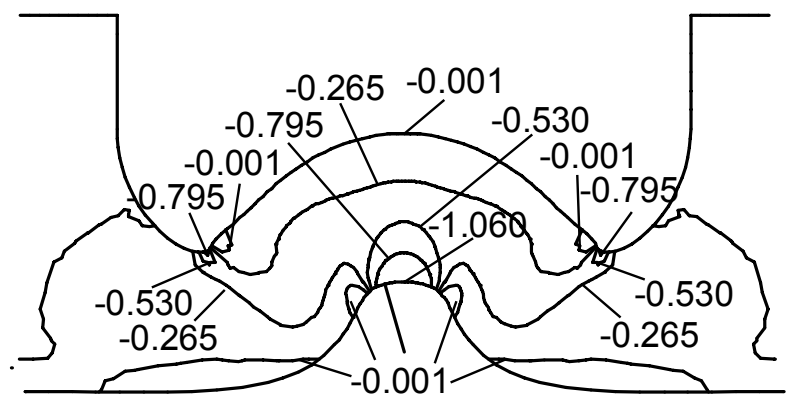

(c) $t=0.8 \mathrm{~mm}$

Fig. 14 Distribution of $\varepsilon_{z}(R=1.0 \mathrm{~mm}, L=5.8 \mathrm{~mm})$

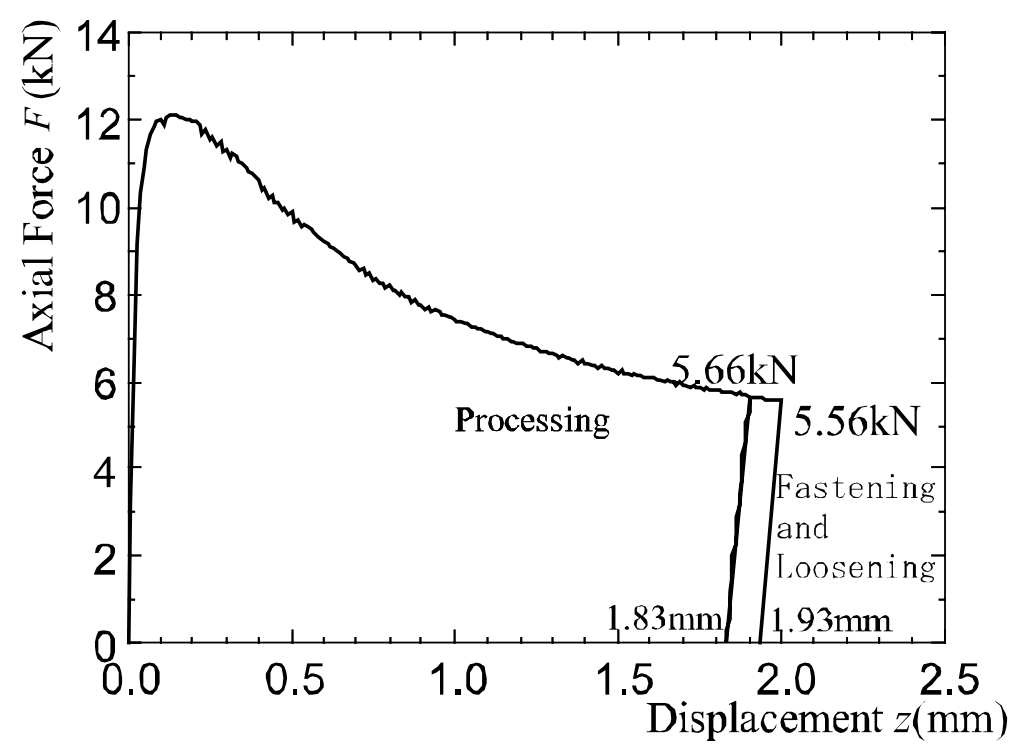

Fig. 15 Relation between axial force and displacement ( $R=0.5 \mathrm{~mm}, L=4.8 \mathrm{~mm}, t=0.4 \mathrm{~mm})$ 


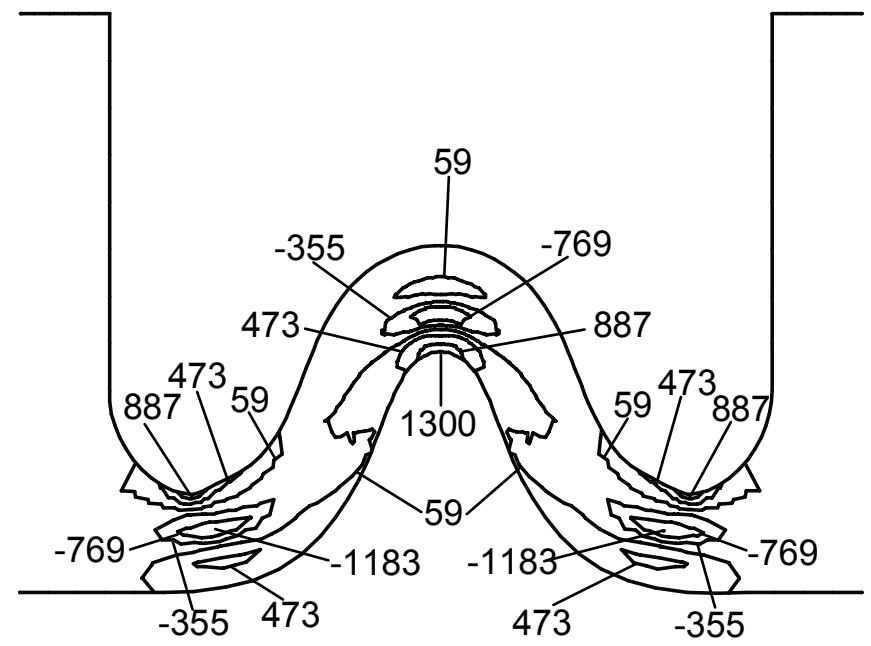

Fig. 16 Distribution of $\sigma_{z}(\mathrm{MPa})$ ( $R=0.5 \mathrm{~mm}, L=4.8 \mathrm{~mm}, t=0.4 \mathrm{~mm})$

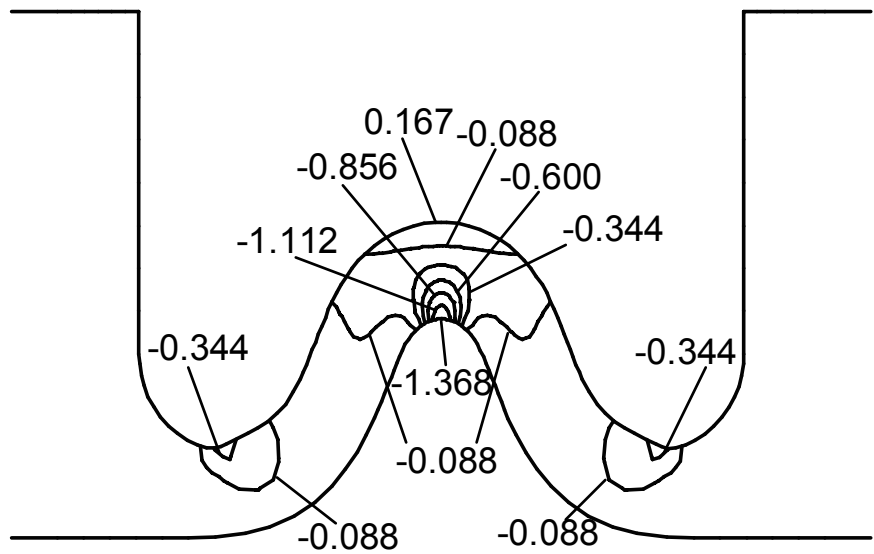

Fig. 17 Distribution of $\varepsilon_{z}$ $(R=0.5 \mathrm{~mm}, L=4.8 \mathrm{~mm}, t=0.4 \mathrm{~mm})$

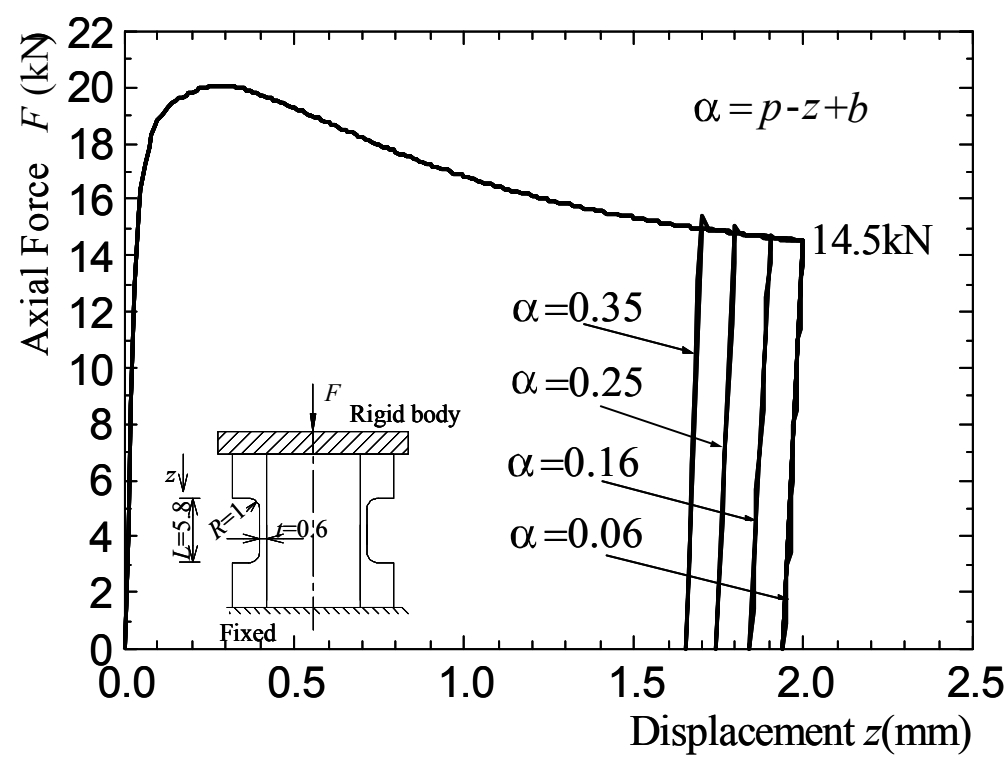

Fig. 18 Relation between axial force and displacement under various phase differences 
With increasing phase difference between lower and upper threads $\alpha$, the plastic deformation becomes larger when the nut is fastened. Accordingly, considering the error in the course of processing, it may be concluded that the phase difference of lower and upper threads should be designed $0.1 \mathrm{~mm}$.

\section{Conclusions}

In this study, "Super Stud Bolt (SSB)" which can prevent self-loosening effectively was studied with the application of FEM. The courses of processing and fasteningloosening were simulated, and the relations between the axial force and displacement were discussed. Also the anti-loosening capability was studied through an axi-symmetric FE model. On the basis of the elastic-plastic large deformation theory and experimental results, the following conclusions can be drawn:

(1) The effect of curvature radius $R$ is investigated when $L$ and $t$ are fixed as $L=$ $5.8 \mathrm{~mm}, t=0.6 \mathrm{~mm}$. It is found that the curvature radius has little effect on the anti-loosening capability and spring constant. It is concluded that the optimum radius curve is $R=0.5 \mathrm{~mm}$ to reduce the maximum stress.

(2) The effect of length $L$ is investigated when dimensions $R$ and $t$ are fixed as $R=$ $0.5 \mathrm{~mm}, t=0.6 \mathrm{~mm}$, and $L$ is changed from $4.8 \mathrm{~mm}$ to $6.8 \mathrm{~mm}$. Large plastic deformation always exists in the course of first fastening-loosening whatever the length $L$ is. It is concluded that the length of thin walled tube should be designed as short as possible if the thin walled tube can be deformed as show in Fig. 5 and therefore the best length is $4.8 \mathrm{~mm}$.

(3) The effect of thickness $t$ is investigated while the $L$ and $R$ are fixed and thickness $t$ is changed as $0.4,0.6$, and $0.8 \mathrm{~mm}$. It is concluded that elasticity of thin walled tube is better when the thickness becomes thinner. Therefore the best $t=0.4 \mathrm{~mm}$ if the strength is enough.

(4) The dimensions of the thin walled tube are optimized as $R=0.5 \mathrm{~mm}, L=4.8 \mathrm{~mm}$, and $t=0.4 \mathrm{~mm}$ considering the anti-loosening capability, spring constant, stress distribution, and cost of processing.

(5) Phase difference has little effect on anti-loosening capability. It is concluded that the optimum phase difference is design $\alpha=0.1 \mathrm{~mm}$ according that plastic deformation is small at the course of fastening.

\section{References}

(1) Daiki Kogyo KK., 2004, Super Slit Nut, Japan Patent (in Japanese) 2004-218674.

(2) Hard Lock Kogyo KK., 2002, Hard Lock Nut, Japan Patent (in Japanese) 2002-195236.

(3) Minamida KK., 1999, Anti-loosening Nut, Japan Patent (in Japanese) H11-177902.

(4) Hard Lock Kogyo KK., 1999, Locking Nut, Japan Patent (in Japanese) H11-006516

(5) Daiki Kogyo KK., 2004, Super Stud Bolt, Japan Patent (in Japanese) 2004-3585.

(6) Kaneta, M., Yamamoto, U., Foundational Mechanical Design Engineering, Rikogakusha, (2003), (in Japanese), pp.58-59.

(7) Nagawa, M., Noda, N.-A., Ichihara, H., Yamada, M., Saito, K., Study of Anti-Loosening Super-Lock Nut and Super-Lock Bolt, Mechanical Design, Vol. 47, No.8, (2003),(in Japanese), pp.35-42 .

(8) Daiki Kogyo KK., 2004, Super Lock Nut, Japan Patent (in Japanese) 2004-3587.

(9) Xiao, Y., Kuhara, M., Noda, N.-A., Saito, K., Nagawa, M., Yumoto, A., Ogasawara, A., Influence of the Shape of Thin Walled Tube on the Mechanical Performance of Super Stud Bolt, Proceedings of the Japan Society of Mechanical Engineers, No.078-1, (2007), pp.23-24.

(10) http://www.superbn.jp/ssb.html. Accessed 2009-11-15. 